\title{
A case of hemichorea following administration of the Pfizer-BioNTech COVID-19 vaccine
}

\author{
Dong-Woo Ryu ${ }^{1}$ (D) Eun-Ye Lim ${ }^{1} \cdot$ A-Hyun Cho ${ }^{1}$
}

Received: 7 October 2021 / Accepted: 16 November 2021 / Published online: 23 November 2021

(c) Fondazione Società Italiana di Neurologia 2021

\section{Dear Editor,}

Hemichorea is a rare condition presenting involuntary hyperkinetic movement on the unilateral side and is primarily caused by structural damage in the basal ganglia or subthalamic nucleus. Some cases of hemichorea have been caused by cortical or thalamic lesions [1]. Though the structural damage is induced mainly by a vascular lesion, hemichorea can develop from other causes including infection, inflammation, and metabolic disease. As many people have received vaccines against coronavirus disease 2019 (COVID-19), various adverse effects (AE) have been reported. The Pfizer-BioNTech COVID-19 vaccine, a messenger RNA (mRNA) vector vaccine, is the only vaccine approved by the U.S. Food and Drug Administration (FDA) and has fewer AEs than vaccines with other platform technologies [2]. However, inflammatory diseases including myocarditis, pancreatitis, and lymphadenopathy and various symptoms have been reported after administration of the Pfizer-BioNTech vaccine [3]. We present a patient who developed hemichorea after administration of the PfizerBioNTech vaccine.

An 83-year-old male was referred for neurologic evaluation due to involuntary hyperkinetic movement of his right arm and leg for 1 month. It was slow, irregular, and purposeless movement and had occurred within 1 day after administration of the second dose of the Pfizer-BioNTech COVID19 vaccine (supplementary video). He had developed no AE after the first dose of the vaccine at 3 weeks prior to the second. His cranial nerve functions, motor power, sensory and cerebellar functions were unremarkable. He denied other symptoms including fever, headache, and myalgia and had

Dong-Woo Ryu

manrew@catholic.ac.kr

1 Department of Neurology, Yeouido St. Mary's Hospital, College of Medicine, The Catholic University of Korea, 10, 63-ro, Yeongdeungpo-gu, Seoul 07345, Republic of Korea neither DM nor history of stroke, although he did take some medicines for hypertension and benign prostate hyperplasia. Brain magnetic resonance imaging (MRI) performed one and half months later from the onset of the symptom showed normal findings except mild stenosis of the middle cerebral and basilar arteries (Fig. 1A). Brain SPECT demonstrated the perfusion pattern asymmetrically decreased in left thalamus (Fig. 1B). Laboratory tests for anti-streptolysin-O, serum cooper, serum ceruloplasmin, vitamin B12, folate, anti-nuclear antibody, anti-phospholipid antibody, $\mathrm{C} 3$ and $\mathrm{C} 4$ complements, and RA factor were normal, as were $\mathrm{HbA} 1 \mathrm{C}(5.6 \%)$, D-dimer $(0.61 \mathrm{mg} / \mathrm{L})$, and prothrombin time INR $(1,04)$. Electroencephalography showed symmetric brain activity without abnormal activity. We started haloperidol $0.75 \mathrm{mg}$ two times a day, and his symptom was mostly relieved at two weeks of follow up.

The Pfizer-BioNTech COVID-19 vaccine can impart immunity against severe acute respiratory syndrome coronavirus 2 (SARS-CoV-2) using an mRNA vector to deliver the genetic code of the antigen to a host. AEs of the PfizerBioNTech COVID-19 vaccine have been reported less frequently and at less severe levels than in vaccines with other technical platforms [2]. Most AEs with the PfizerBioNTech COVID-19 vaccine are simple local reactions and systemic reactions including fever, myalgia, and headache. Serious AEs, such as myocarditis, lymphadenopathy, and anaphylaxis, have been reported in rare cases. Neurological AEs that developed after administration of the Pfizer-BioNTech COVID-19 vaccine were tingling, numbness, limb weakness, vertigo, seizure, and loss of consciousness. Bell's palsy and Guillain-Barre syndrome were reported in some cases [3, 4]. Brain structural damage has been rarely reported after the Pfizer-BioNTech COVID-19 vaccine. Although further studies are in process to understand the mechanism of $\mathrm{AE}$ in the PfizerBioNTech COVID-19 vaccine, it is supposed that inflammation or infection in multiple organs including those of the nervous system could occur after the vaccination. 


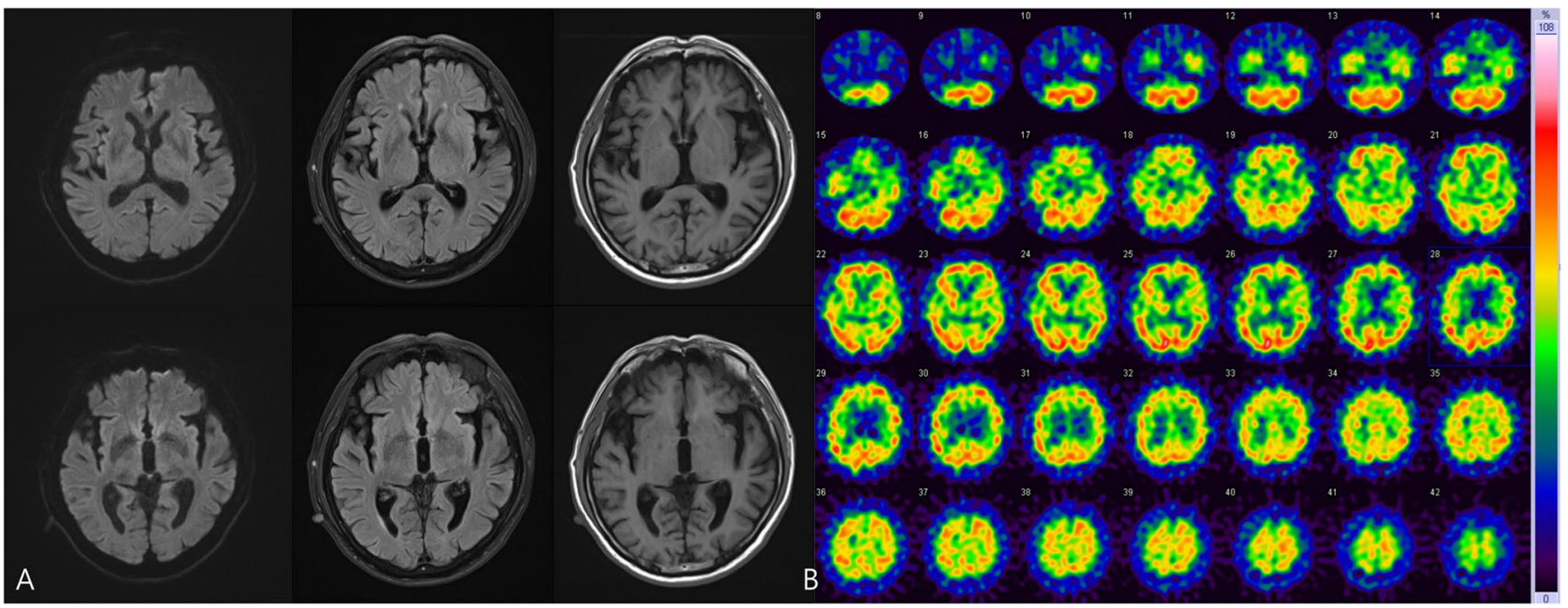

Fig. 1 Brain MRI results of the patient (A). Diffusion-weighted, fluid-attenuated inversion recovery, and T1-weighted axial images show normal findings in the basal ganglia, thalamus, and surrounding brain regions. Brain SPECT (B) showed left thalamic hypoperfusion compared with right side
Headache and severe AEs have been reported with myocarditis and anaphylaxis.

The pathophysiology of hemichorea in this case is unclear. Acute onset of the hyperkinetic movement on the unilateral side supposes that it is caused by structural damage in the brain. Brain SPECT revealed contralateral thalamic hypoperfusion, which can be concerned with hemichorea. Although no structural damage was found on brain MRI, a small lesion can be concealed between slices $4-\mathrm{mm}$ thick. In addition, metabolic disturbances induced by the Pfizer-BioNTech COVID-19 vaccine could affect the thalamus, which contains the regulatory pathway for movement. Thalamic dysfunction can disrupt important connections for the coordination of movement and develop aberrant pathway which can evoke abnormal involuntary movements. In ChAdOx1 adeno-virus vector vaccine, sudden increasing serum glucose level following vaccination has been reported in some cases [5]. In addition, other systemic illness may induce embolism, which can cause thalamic dysfunction or hypoperfusion.

To the best of our knowledge, this is a first report of hemichorea following administration of the PfizerBioNTech COVID-19 vaccine. Although brain MRI, electroencephalography, and laboratory test showed no causative abnormality in this report, functional disturbance was found in contralateral thalamus using SPECT. His symptom was a typical aspect of hemichorea and developed suddenly in temporal association with the vaccination. Therefore, it is supposed that the PfizerBioNTech COVID-19 vaccine can be cause of hemichorea by an interruption in the unilateral thalamic function. Due to the possibility that other factors could affect the development of hemichorea in this case, we planned to evaluate for follow up. Further studies and more reports like ours are needed to expand the knowledge of hemichorea and COVID-19 vaccines.

Supplementary Information The online version contains supplementary material available at https://doi.org/10.1007/ s10072-021-05763-5.

\section{Declarations}

Ethical approval None.

Conflict of interest The authors declare no competing interests.

\section{References}

1. Li ZS, Fang JJ, Xiang XH, Zhao GH (2021) Hemichorea due to ipsilateral thalamic infarction: a case report. World J Clin Cases 9(19):5287-5293. https://doi.org/10.12998/wjcc.v9.i19.5287

2. Menni C, Klaser K, May A et al (2021) Vaccine side-effects and SARS-CoV-2 infection after vaccination in users of the COVID Symptom Study app in the UK: a prospective observational study. Lancet Infect Dis 21(7):939-949. https://doi.org/10.1016/ s1473-3099(21)00224-3 
3. Kadali RAK, Janagama R, Peruru S, Malayala SV (2021) Side effects of BNT162b2 mRNA COVID-19 vaccine: a randomized, cross-sectional study with detailed self-reported symptoms from healthcare workers. Int J Infect Dis 106:376-381. https://doi. org/10.1016/j.ijid.2021.04.047

4. Scendoni R, Petrelli C, Scaloni G, Logullo FO (2021) Electromyoneurography and laboratory findings in a case of GuillainBarré syndrome after second dose of Pfizer COVID-19 vaccine. Hum Vaccin Immunother:1-4. https://doi.org/10.1080/21645 515.2021 .1954826
5. Mishra A, Ghosh A, Dutta K, Tyagi K, Misra A (2021) Exacerbation of hyperglycemia in patients with type 2 diabetes after vaccination for COVID19: report of three cases. Diabetes Metab Syndr 15(4):102151. https://doi.org/10.1016/j.dsx.2021.05.024

Publisher's note Springer Nature remains neutral with regard to jurisdictional claims in published maps and institutional affiliations. 